\title{
Review of LEP Results
}

\author{
F. Parodi representing the LEP collaborations
}

Dipartimento di Fisica, Via Dodecaneso 33, 16146 Genova, Italy

\begin{abstract}
I present a review of the results obtained during 10 years of activity in $b$-physics at LEP. Special emphasis will be put on measurements that attained precisions not even envisaged at the beginning of the LEP programme $\left(V_{u b}\right.$ and $\left.\Delta m_{s}\right)$. Finally the impact of these measurements on the CKM parameters determination will be presented.
\end{abstract}

\section{INTRODUCTION}

The $b$-physics program at LEP have covered different domains. In this paper I will concentrate on the measurements related to determination of the parameters of the CKM matrix, neglecting, because of lack of space, electroweak and spectroscopy results.

The leading role of LEP in the last decade of $b$-physics is evident not only in the experimental results but also in the averaging activities. LEP Working Groups have pushed LEP, SLD and Tevatron communities to compare results and to invent new methods for combining measurements in order to exploit at best the available data.

This work should be continued by new experiments.

Most of the results presented here comes, except where explicitly stated, from the summary paper produced by the LEP Heavy Flavour Working Groups for Summer Conferences 2001 [1].

\section{B-LIFETIME MEASUREMENTS}

The measurements of average lifetime of weakly decaying B-hadrons are an important test of the $B$-decay dynamics. In the naive spectator model all the $B$ lifetimes should be equal the contribution of other processes (exchange, annihilation) contributing to the total decay rate modify this simple picture.

The Operator Product Expansion, within the factorization approximation, give, for the lifetime ratio, the following predictions [2]:

$$
\frac{\tau\left(B^{+}\right)}{\tau\left(B_{d}^{0}\right)}=1+0.05 \times \frac{f_{B}^{2}}{(200 M e V)^{2}}, \frac{\tau\left(B_{s}^{0}\right)}{\tau\left(B_{d}^{0}\right)}=1 \pm O(1 \%), \frac{\tau\left(\Lambda_{b}^{0}\right)}{\tau\left(B_{d}^{0}\right)}=0.90-0.95
$$

The study of the $B$-lifetimes has been also stimulating new methods for selecting pure samples of a definite $B$ specie. At the beginning of LEP the first signal of $B_{s}^{0}$ and $\Lambda_{b}^{0}$ have been selected looking for right sign correlations, in the same hemisphere of a selected event, between the sign of the lepton issued by a semi-leptonic decay and the accom-

CP618, Heavy Flavor Physics: Ninth International Symposium, edited by A. Ryd and F. C. Porter (c) 2002 American Institute of Physics 0-7354-0064-4/02/\$19.00

32 
panying charm hadron. Afterwards semi-inclusive reconstructions of the charm hadron have been used to increase the available statistics and finally, in the last years, charged and neutral $B$ hadrons have been separated efficiently using neural network methods. The average lifetimes from LEP/SLD/Tevatron computed for the 2001 Summer Conference $^{1}[3]$ show remarkable precisions:

$$
\begin{aligned}
& \tau\left(B_{d}^{0}\right)=1.545 \pm 0.020 \mathrm{ps} \\
& \tau\left(B^{+}\right)=1.642 \pm 0.017 \mathrm{ps} \\
& \tau\left(\mathrm{B}_{\mathrm{s}}^{0}\right)=1.464 \pm 0.057 \mathrm{ps} \\
& \tau\left(\Lambda_{b}\right)=1.208 \pm 0.051 \mathrm{ps} \\
&
\end{aligned}
$$

Comparing these results with the predictions of Equation 1 one can conclude that:

- the ratio $\tau\left(B^{+}\right) / \tau\left(B_{d}^{0}\right)$ show a $3 \sigma$ deviation from unity in agreement with theory;

- the ratio $\tau\left(B_{s}^{0}\right) / \tau\left(B_{d}^{0}\right)$ is in agreement with theory;

- the measurements of the $\Lambda_{b}^{0}$ lifetime are already precise enough to spot an inconsistency with the prediction and push for a better understanding of the theory.

The experimental results show that the hierarchy has been correctly predicted by theory. More stringent tests will be performed by $B$-factories and Tevatron.

\section{LIFETIME DIFFERENCE: $\Delta \Gamma_{S}$}

The lifetime difference between the weak eigenstates in the $B_{s}^{0}-\bar{B}_{s}^{0}$ system is expected to be small, to the first approximation $\Delta \Gamma_{s} / \Delta m_{s} \simeq 3 / 2 \pi\left(m_{b} / m_{t}\right)^{2}$ [4]. Theoretical calculations [5] of the ratio $\Delta \Gamma_{s} / \Gamma_{s}$ at next-to-leading order give:

$$
\left.\frac{\Delta \Gamma_{s}}{\Gamma_{s}}=\left(\frac{f_{B_{s}}}{230 \mathrm{MeV}}\right)^{2} 0.007 B_{b}\left(m_{b}\right)+\left(0.132_{0.027}^{0.011}\right) \bar{B}_{s}\left(m_{b}\right)-(0.078 \pm 0.018)\right]
$$

where $f_{B_{s}}$ is the $B_{s}^{0}$ decay constant, $B_{b}\left(m_{b}\right)$ and $\bar{B}_{b}\left(m_{b}\right)$ are bag parameters.

The study of $\Delta \Gamma_{s}$ benefit from the work done on lifetimes. Experimental informations on $\Delta \Gamma_{s}$ have been extracted by studying the proper time distribution of data samples enriched in $B_{s}$ mesons or by measuring the branching fraction $B_{s} \rightarrow D_{s}^{(*)+} D_{s}^{(*)-}$.

In order to obtain an improved limit on $\Delta \Gamma_{s}$, the results based on fits to the proper time distributions are used to constrain $1 / \Gamma_{s}$ to the world average lifetime $\tau\left(B_{d}^{0}\right)$. This is well motivated theoretically, as the total widths of the $B_{s}^{0}$ and $B_{d}^{0}$ mesons are expected to be equal within less than one percent and $\Delta \Gamma_{d}$ is expected to be small.

The combined results from LEP/Tevatron on $\Delta \Gamma_{s}$ are:

$$
\begin{array}{cc}
\text { Assuming } \tau\left(B^{0}\right)=\tau\left(\mathrm{B}_{\mathrm{s}}^{0}\right) & \text { No assumptions } \\
\Delta \Gamma_{s} / \Gamma_{s}=0.16_{-0.09}^{+0.08} & \Delta \Gamma_{s} / \Gamma_{s}=0.24_{-0.12}^{+0.16} \\
\Delta \Gamma_{s} / \Gamma_{s}<0.3195 \% \text { C.L. } & \Delta \Gamma_{s} / \Gamma_{s}<0.5395 \% \text { C.L. }
\end{array}
$$

\footnotetext{
${ }^{1}$ This average includes also BaBar measurements
} 
The possibility of using these combined limits for the indirect determination of $\Delta m_{s}$ is still limited by the theoretical uncertainties in the evaluation of $\Delta \Gamma_{s} / \Delta m_{s}[6]$.

$$
N_{C} \text { vs. } B R(B \rightarrow \ell v)
$$

The measured value of the $b$-hadron semileptonic decay branching fraction is, since several years, on the low side of the theoretical expectations [7]. One way to reconcile theory with experiments consists in assuming that the $c$-quark effective mass is lower than used in these evaluations; this implies that decays of the type $b \rightarrow c \bar{c} s(d)$ correspond to larger decay rate. The average number of $c$ and $\bar{c}$ quarks contributing in $b$-hadron decays is thus negatively correlated with the expected value of $B R(b \rightarrow \ell X)$ : the simultaneous measurement of these two quantities may help to clarify the theoretical picture.

Experimentally, the number of $c$ and $\bar{c}$ quarks contributing to $b$-hadron decays can be obtained by measuring the production fractions of charmed hadrons and charmonium states. Measurements originate from four sources:

- open-charm counting using exclusively reconstructed charmed hadrons,

- charmonium production,

- inclusive measurements of the distribution of charged track allowing the determination of $b \rightarrow D \bar{D} X$ and $b \rightarrow 0 D$,

- $b \rightarrow D_{i} \bar{D}_{j} X$ branching fraction measurements in which $D_{i(j)}$ are completely reconstructed.

Measurements done at $\Upsilon(4 S)$ and at the $\mathrm{Z}$ have been combined separately and they are showing in Figure 1. These results, summarizing 10 years of experimental activity, are

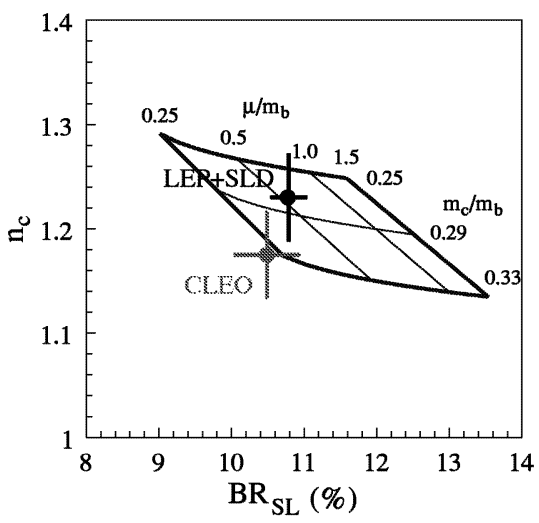

FIGURE 1. Comparison between the measured number of $c$ and $\bar{c}$ quarks in $b$-hadron decays and of inclusive semileptonic fraction, with theoretical predictions.

compatible with the theoretical predictions and favour a rather standard value for the charm quark mass and a low scale, $\mu$, at which QCD corrections have to be evaluated. 


\section{$\left|V_{C B}\right|$}

$\left|V_{c b}\right|$ can be determined with two methods: the inclusive method, which uses semileptonic decay width of $b$-decays and the OPE, and the exclusive method, where $\left|V_{c b}\right|$ is extracted by studying the exclusive $\bar{B}_{d}^{0} \rightarrow D^{*+} \ell^{-} \bar{v}_{\ell}$ decay process using HQET (measuring the product $\left.\mathcal{F}(1)\left|V_{c b}\right|\right)$.

Measurements done at LEP give, using the inclusive method:

$$
\left|V_{c b}\right|^{\text {incl. }}=(40.7 \pm 0.5(\text { exp. }) \pm 2.4(\text { theo. })) \times 10^{-3}
$$

and using the exclusive method with $\mathcal{F}(1)=0.88 \pm 0.05$ [8]:

$$
\left|V_{c b}\right|^{\text {incl. }}=(40.5 \pm 1.9(\text { exp. }) \pm 2.3(\text { theo. })) \times 10^{-3}
$$

The LEP $\left|V_{c b}\right|$ Working Group computed a combined average taking into account the correlations between the two methods. The combined value is:

$$
\left|V_{c b}\right|=(40.6 \pm 1.9) \times 10^{-3}
$$

where $1.0 \times 10^{-3}$ comes from correlated sources.

$$
\left|V_{U B}\right|
$$

The LEP Collaborations have measured, using different techniques, the inclusive yield of $b \rightarrow u$ transitions in semi-leptonic $B$ decays. ALEPH and OPAL used a neural network discriminant based in kinematical variables, DELPHI preferred a classification based on the reconstructed mass $M_{X}$, decay topology and presence of secondary kaons, while L3 adopted a sequential cut analysis based on the kinematics of the two leading hadrons produced in the same hemisphere as a tagged lepton.

Each experiment optimize the performance of its analysis by choosing different working points of efficiency and signal-to-background ratio $(S / B)$. Starting from a natural $S / B$ of $\simeq 0.02$, ALEPH obtained $S / B=0.07$ with an efficiency $\varepsilon=11 \%$, DELPHI had $S / B=0.10$ with $\varepsilon=6.5 \%$ and $\mathrm{L} 3 S / B=0.16$ with $\varepsilon=1.5 \%$.

Figure 2 show the evidence of inclusive charmless decays in a recent analysis by OPAL [9].

The LEP average give [10]:

$$
B R\left(b \rightarrow \ell^{-} \vee X_{u}\right)=(1.67 \pm 0.31(\text { stat. }+ \text { exp. }) \pm 0.37(b \rightarrow c) \pm 0.2(b \rightarrow u)) \times 10^{-3}
$$

The magnitude of the matrix element $V_{u b}$ has been extracted using the following relationship derived in the context of OPE [11]:

$$
\left|V_{u b}\right|=0.00445 \sqrt{\frac{B R\left(b \rightarrow X_{u} \ell^{-} \overline{\mathrm{v}}_{\ell}\right) 1.55 p s}{0.002 \tau_{b}}} \times\left(1 \pm 0.010(\text { pert }) \pm 0.030\left(1 / m_{b}^{5}\right) \pm 0.035\left(m_{b}\right)\right)
$$



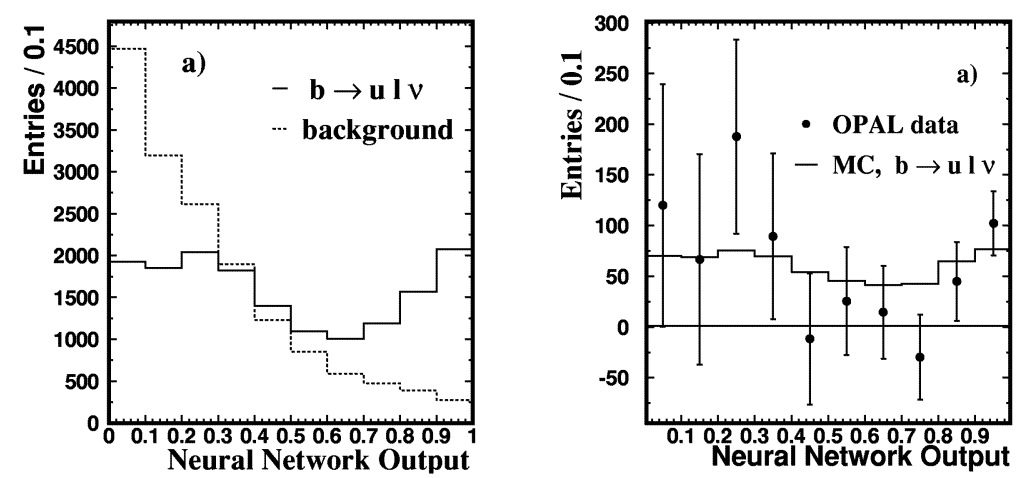

FIGURE 2. OPAL Collaboration: evidence of charmless decays as a function of the neural network output.

by assuming $m_{b}=(4.58 \pm 0.06) \mathrm{GeV} / \mathrm{c}^{2}$; this give

$$
\left|V_{u b}\right|=(40.5 \pm 6.2(\exp .) \pm 3.1(\text { theo. })) \times 10^{-4}
$$

This result is compatible and competitive with the determination based on the decays $B \rightarrow \rho \ell v$ :

$$
\left|V_{u b}\right|=(32.5 \pm 2.9(\exp .) \pm 5.5(\text { theo. })) \times 10^{-4}
$$

The two determinations are also complementary since the theoretically uncertainties are totally uncorrelated.

\section{$B^{0}-\overline{B^{0}}$ OSCILLATIONS}

The oscillations between particle and anti-particle in neutral B mesons systems is a process at the second order in the electroweak perturbation theory. The frequency of this oscillation is given by the mass difference between the two weak eigenstates:

$$
\Delta m_{q} \propto m_{W}^{2} f_{B_{q}}^{2} B_{B_{q}}\left|V_{t q}\right|^{2}\left|V_{t b}\right|^{2}
$$

where $f_{B_{q}}^{2} B_{B_{q}}$ is a non-perturbative QCD parameter and $V_{i j}$ are parameters of the CKM matrix. The hierarchy of CKM parameters makes $\Delta m_{s} \approx 20 \Delta m_{d}$ and consequently the $B_{s}^{0}$ oscillations difficult to observe.

From the theoretical side it is important to measure precisely both $\Delta m_{d}$ and $\Delta m_{s}$ because in the ratio $\Delta m_{d} / \Delta m_{s}$ the non perturbative QCD part is believed to be better under control than in the absolute values. 


\section{$B_{d}^{0}-\overline{B_{d}^{0}}$ system}

The time depend study of $B_{d}^{0}$ oscillations has been pioneered at LEP. The precision of world average of $\Delta m_{d}$ receives, at present, similar contributions by B-factories and LEP/SLD/Tevatron:

$$
\begin{aligned}
\Delta m_{d}(L E P / S L D / C D F) & =(0.492 \pm 0.013) p^{-1} \\
\Delta m_{d}(B-\text { Factories }) & =(0.485 \pm 0.010) p^{-1} \\
\Delta m_{d}(\text { World avg. }) & =(0.489 \pm 0.008) p s^{-1} \\
B_{s}^{0}-\overline{B_{s}^{0}} & \text { system }
\end{aligned}
$$

The study of the $B_{s}^{0}$ oscillations requires, because of the expected high value of $\Delta m_{s}$, good proper time resolution and clean samples of $B_{s}^{0}$ decays.

At LEP/SLD/Tevatron in the last few years several analyses have been tried ranging from the most inclusive (several 10000 events) with low $B_{s}$ purity $(\simeq 10 \%)$ to the most exclusive (few 100 events) with high $B_{s}$ purity and high proper time resolution. Moreover, in most of the analyses, discrimant methods have been developed in order to separated the bulk of precisely measured candidates from the rest of the sample.

No experiment has yet directly observed $B_{s}^{0}$ oscillations but the sensitivity of the combined world average analysis has improved over the years.

The combination is performed, by the LEP Oscillations Working Group, in the framework of the amplitude method [12]. At each value of $\Delta m_{s}$ an amplitude is measured in each analysis, where the expected value of the amplitude is unity at the true frequency. An overall limit on $\Delta m_{s}$ is then inferred from the combined amplitude spectrum by excluding regions where the amplitude is incompatible with unity. The sensitivity of the analisys is defined as the limit one would get if all the amplitude values were put at 0 . The present world average give the lower limit

$$
\Delta m_{s}>14.8 p s^{-1} @ 95 \% \text { C.L. } \text { with a sensitivity of } \Delta m_{s}=18 p s^{-1}
$$

\section{How to use $\Delta m_{s}$ in CKM fits?}

The amplitude spectrum contains more information than the 95\%C.L. limit. In the present case, for instance, there is a "hint" of a signal at $17 \mathrm{ps}^{-1}$ with $2.6 \sigma$ significance. The matter is not to decide if this is a signal or not ${ }^{2}$ but to use the information coming from data without introducing any bias.

\footnotetext{
${ }^{2}$ The $3 \sigma$ or $5 \sigma$ criteria are only useful conventions.
} 

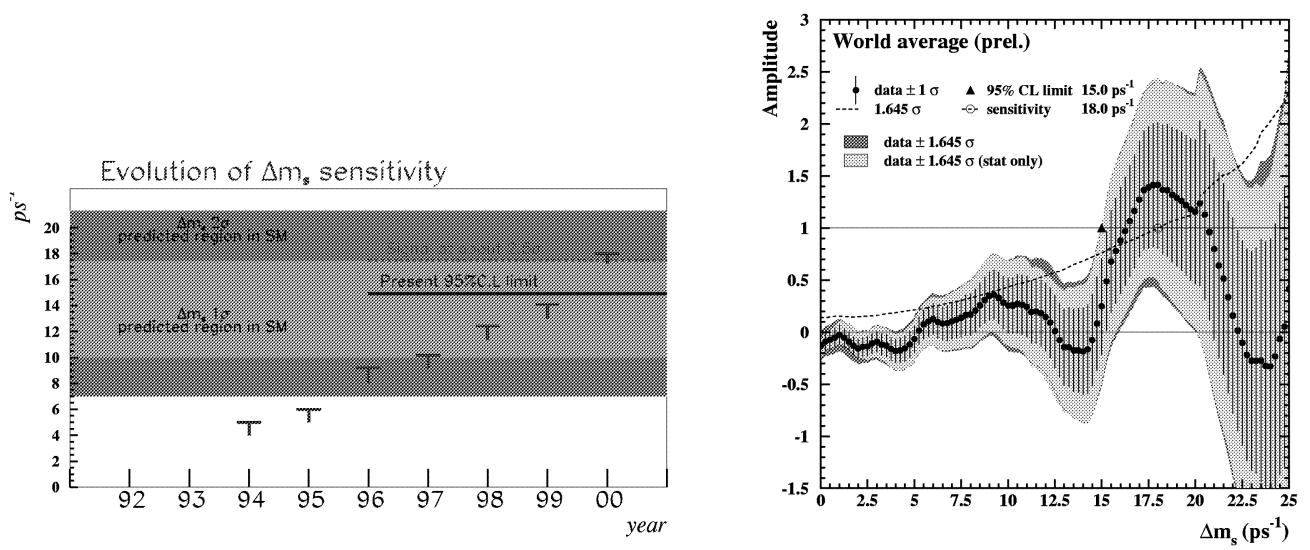

FIGURE 3. a) Evolution of the $\Delta m_{s}$ sensitivity. b) World average amplitude spectrum

\section{Likelihood ratio $R$ method}

Recently it has been proposed to use the log-likelihood function $\Delta \log \mathcal{L}^{\infty}\left(\Delta m_{s}\right)$ referenced to its value obtained for $\Delta m_{s}=\infty$ [13]. Similar considerations, developed in a different context, have been detailed in [14]. The log-likelihood values can be easily deduced from $\mathcal{A}$ and $\sigma_{\mathcal{A}}$ using the expressions given in [12]; the likelihood ratio $\mathrm{R}$ is then defined as:

$$
\mathrm{R}\left(\Delta m_{s}\right)=e^{-\Delta \log \mathcal{L}^{\infty}\left(\Delta m_{s}\right)}=\frac{\mathcal{L}\left(\Delta m_{s}\right)}{\mathcal{L}\left(\Delta m_{s}=\infty\right)} .
$$

The function $\mathrm{R}$ corresponds to the ratio of the probability density for a given $\Delta m_{s}$ value over the probability density for $\Delta m_{s}=\infty$.

\section{Modified $\chi^{2}$ method}

In the first CKM fits $\chi^{2}$ with respect to 1 was used:

$$
\chi^{2}=\left(\frac{1-\mathcal{A}}{\sigma_{\mathcal{A}}}\right)^{2}
$$

This method has two main drawbacks:

- the sign of the deviation of the amplitude with respect to the value $\mathcal{A}=1$ was not used, whereas it is expected that an evidence for a signal would manifest itself by giving an amplitude value which is simultaneously compatible with $\mathcal{A}=1$ and incompatible with $\mathcal{A}=0$;

- values of $\mathcal{A}>1$ are disfavoured w.r.t. $\mathcal{A}=0$, while it is expected that, because of statistical fluctuations, the amplitude value corresponding to the "true" $\Delta m_{s}$ value 
could be higher than 1 . This problem was solved in the early days of the use of $\Delta m_{s}$ in CKM fits putting $\mathcal{A}=1$ whenever higher.

In [15] the $\chi^{2}$ has been modified ad hoc to solve the second problem:

$$
\chi^{2}=2 \cdot\left[\operatorname{Erfc}^{-1}\left(\frac{1}{2} \operatorname{Erfc}\left(\frac{1-A}{\sqrt{2} \sigma_{A}}\right)\right)\right]^{2}
$$

\section{Comparison of the two methods}

The amplitude as a function of $\Delta m_{s}$ and the corresponding $\Delta \log \mathcal{L}\left(\Delta m_{s}\right)$ are shown in Figure 4-a),b). The corresponding Likelihoods obtained using the Likelihood ratio and the Modified $\chi^{2}$ methods are shown in Figure 4-c). It is clear that the two methods give
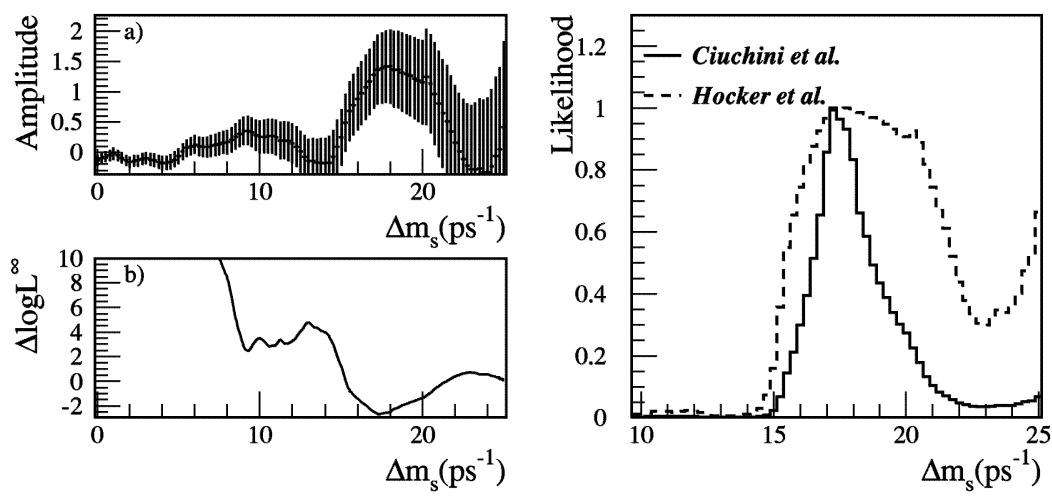

FIGURE 4. World average amplitude analysis: a) amplitude spectrum, b) $\Delta \log \mathcal{L}^{\infty}\left(\Delta m_{s}\right)$, c) comparison between $\mathrm{R} \equiv$ Likelihood ratio method and the Modified $\chi^{2}$ method.

very different Likelihood functions. In particular the Modified $\chi^{2}$ method give a less tight constraint.

However no conclusion can be deduced from a single experiment.

The minimal requirement for a "good" method is to give the correct probability density function in case of an oscillation signal. To test this case a toy Montecarlo has been generated with $\Delta m_{s}=17 p s^{-1}$ with an average significance at that value of $6 \sigma$.

The results are shown in Figure 5. It is clear that the Likelihood ratio method is able to see the signal at the correct $\Delta m_{s}$ value, whereas the Modified $\chi^{2}$ method failed. The same exercise was repeated at different generated values of $\Delta m_{s}$ giving similar results.

Only the Likelihood ratio method will be then used in the following.

\section{UNITARITY TRIANGLE FIT}

The unitarity triangle fit presented here is based on the Bayesian approach described in [16]. The constraint are $\left|V_{u b}\right| /\left|V_{c b}\right|, \varepsilon_{K}, \Delta m_{d}$ and $\Delta m_{s}$ (used as described in the previous 

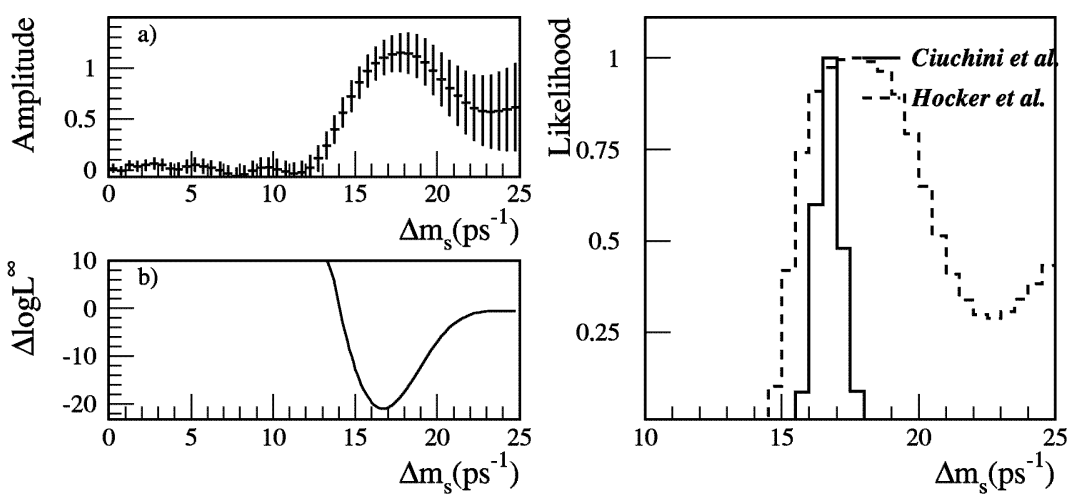

FIGURE 5. Toy-MC analyses with the same $\sigma(\mathcal{A})$ versus $\Delta m_{s}$ behaviour as the world average analysis : a) amplitude spectrum, b) $\Delta \log \mathcal{L}^{\infty}\left(\Delta m_{s}\right)$, c) comparison between $\mathrm{R} \equiv$ Likelihood ratio method and the Modified $\chi^{2}$ method.

section) and the list of parameter of [16] has been updated with the most recent values, presented in this review, of $\left|V_{u b}\right|,\left|V_{c b}\right|$ and $\Delta m_{s}$.

The allowed region in the $\rho-\eta$ plane in the Standard Model framework is shown in Figure 6 . The fitted values of $\bar{\rho}$, of $\bar{\eta}$ and of the angles of unitarity triangle are accurately

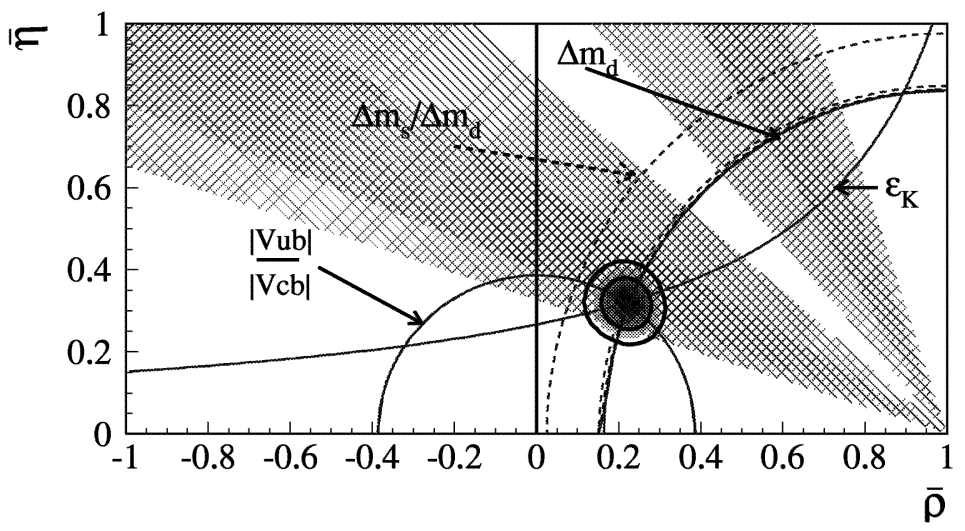

FIGURE 6. Allowed region in the $\bar{\rho}-\bar{\eta}$ plane selected by the $\left|V_{u b}\right| /\left|V_{c b}\right|, \varepsilon_{K}, \Delta m_{d}$ and $\Delta m_{s}$ constraints. The $\sin 2 \beta$ constraint, not used in the fit, is superimposed.

determined:

$$
\begin{gathered}
\bar{\rho}=0.218 \pm 0.038 \\
\bar{\eta}=0.316 \pm 0.040 \\
\sin 2 \beta=0.696 \pm 0.067 \\
\sin 2 \alpha=-0.42 \pm 0.23 \\
\gamma=(55.5 \pm 6.2)^{\circ}
\end{gathered}
$$


The indirect determination of $\sin 2 \beta$ is compatible with the world average of $\sin 2 \beta$ (dominated by $\mathrm{BaBar}$ and Belle measurements): $\sin 2 \beta=0.79 \pm 0.10$.

The result of the fit show that the measurements performed at LEP/SLD/Tevatron constrained efficiently the sides of the unitarity triangle allowing the determine, in indirect way, $\sin 2 \beta$ and $\gamma$ at $10 \%$. The comparison between the sides and the angles of unitarity triangle will become more stringent as soon as the $\sin 2 \beta$ measurements will improve and new experimental constraints (other angles, constraints from Kaon physics, ... ) will be added.

\section{ACKNOWLEDGMENTS}

The results presented in the section "Unitarity triangle fit" are based on work done in collaboration with M. Ciuchini, G. D' Agostini, E. Franco, V. Lubicz, G. Martinelli, P. Roudeau, L. Silvestrini and A. Stocchi.

\section{REFERENCES}

1. ALEPH, CDF, DELPHI, L3, OPAL, SLD Collaborations, CERN-EP, 2001-050, 1 (2001),

2. G. Bellini, I.I. Bigi and P.J. Dornan, Phys. Rep., 289, 1 (1997).

3. LEP Lifetime Working Group, Averages for Summer Conferences 2001 (2001), URL http: //claires.home.cern.ch/claires/lepblife.html, and references therein.

4. A. J. Buras, W. Slominski and H. Steger, Nucl. Phys., B284, 369 (1984).

5. M. Beneke et al., Phys. Lett., B459, 631 (1999).

6. D. Becirevic, D. Meloni, A. Retico, V. Gimenez, V. Lubicz and G. Martinelli, Phys. J., C18, 157 (2000).

7. M. Neubert and C.T. Sachrajda, Nucl. Phys., B483, 339 (1997).

8. I.I. Bigi, M. Shifman and N. Uraltsev, Annu. Rev. Nucl. Part. Sci., 47, 591 (1997).

9. The OPAL Collaboration, G. Abbiendi et al., Eur. Phys. J., C21, 399 (2001).

10. The LEP $V_{u b}$ Working Group, LEPVUB, 01/01 (2001).

11. N. Uraltsev et al., Eur. Phys. J., C4, 453 (1998).

12. G. D’Agostini and G. Degrassi, Eur. Phys. J., C10, 633 (1999).

13. P. Checchia, E. Piotto, F. Simonetto, hep-ph, 9907300, 1 (1999).

14. G. D'Agostini, hep-ph, 0002055, 1 (2000).

15. A. Hocker et al., Eur. Phys. J., C21/2, 225 (2001).

16. M. Ciuchini et al., JHEP, 0107, 013 (2001). 